KOBE-TH-97-02

September 1997

\title{
Dynamical symmetry breaking in the external gravitational and constant magnetic fields
}

\author{
T. Inagaki 用, \\ Department of Physics, Kobe University, Rokkoudai, Nada, Kobe 65\%, Japan \\ S. D. Odintsov [7, \\ Dept. Theor. Phys., Tomsk Pedagogical University, 634041 Tomsk, Russia \\ and \\ Department de Fisica, Universidad del Valle, A.A.25360 Cali, Colombia \\ Yu. I. Shil'nov 用 \\ Institut D'Estudis Espacials De Catalunya, \\ Edif. Nexus-104, Gran Capita 2-4, 08034, Barcelona, Spain \\ and \\ Department of Theoretical Physics, Faculty of Physics, \\ Kharkov State University, Svobody Sq. 4, 310077, Kharkov, Ukraine
}

\begin{abstract}
We investigate the effects of the external gravitational and constant magnetic fields to the dynamical symmetry breaking. As simple models of the dynamical symmetry breaking we consider the Nambu-Jona-Lasinio (NJL) model and the supersymmetric Nambu-Jona-Lasinio (SUSY NJL) model non-minimally interacting with the external gravitational field and minimally interacting with constant magnetic field. The explicit expressions for the scalar and spinor Green functions are found up to the linear terms on the spacetime curvature and exactly for a constant magnetic field. We obtain the effective potential of the above models from the Green functions in the magnetic field in curved spacetime. Calculating the effective potential numerically with the varying curvature and/or magnetic fields we show the effects of the external gravitational and magnetic fields to the phase structure of the theories. In particular, increase of the curvature in the spontaneously broken chiral symmetry phase due to the fixed magnetic field makes this phase to be less broken. On the same time strong magnetic field quickly induces chiral symmetry breaking even at the presence of fixed gravitational field within nonbroken phase.
\end{abstract}

${ }^{*}$ e-mail : inagaki@hetsun1.phys.kobe-u.ac.jp

${ }_{\dagger}^{\dagger}$-mail : sergei@ecm.ub.es, odintsov@quantum.univalle.edu.co

${ }^{\ddagger} \mathrm{e}-\mathrm{mail}$ : visit2@ieec.fcr.es 


\section{Introduction}

The idea of the dynamical symmetry breaking in quantum field theory has been introduced quite long ago [1]. In the attempts to realize this idea in the early universe one should study the external gravitational field (or curved spacetime). The investigation of the dynamical symmetry breaking in four-fermion models [1], 2] in four and three

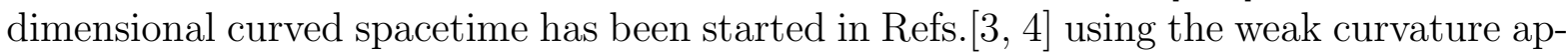
proximation (for a review and list of references, see [5]). A validity of the weak curvature approximation is discussed in Ref.[6]. In the case when the external gravitational field is treated exactly (de Sitter or anti-de Sitter or Einstein universe background) the phase structure of the four-fermion models has been studied in Refs. [7]. (for a renormalization group approach, see also [8]).

There appeared recently some indications that early universe may contain large primordial magnetic fields. The role of these fields in cosmology (in particularly, in the inflationary models of the universe) has been discussed in Refs. [9]. The presence of magnetic field in the early universe may lead to different effects. In particular, it is reasonable then to investigate the dynamical symmetry breaking in curved spacetime with magnetic fields s. Such study for 3D and 4D four-fermion models has been undertaken in Refs. [13, 14] in the approximation when the effects of the gravitational and magnetic field maybe simply summarized (in other words, the correspondent terms in the effective potential are evaluated with the neglecting of the interaction between gravitational and magnetic fields). However, the coherent effect of combined gravitational and magnetic field may become very relevant.

That is the purpose of the present work to investigate the phase structure of fourfermion models in weakly curved spacetime with constant magnetic field. We develop the approximation where the effective potential and Green function (GF) of the theory maybe represented as the expansion in the powers of curvature invariants. In each order of this expansion the external magnetic field is treated exactly, i.e. we take into account the coherent effect of combined gravitational and magnetic fields.

The paper is organized as follows. In the next section we present new form of the local momentum representation of propagators [15] with account of the constant magnetic field. In each order on the expansion in curvature invariants for Green functions the magnetic field is included exactly. The detailed calculation is presented for scalar and spinor Green functions. Section 3 is devoted to the study of phase structure in the NJL model in curved spacetime with magnetic field. We calculate the effective potential with account of linear curvature terms for $3 \mathrm{D}$ and $4 \mathrm{D}$ models. The numerical investigation of the effective potential for 4D NJL model is given. In section 4 we discuss the SUSY NJL model non-minimally interacting with the external gravitational field and minimally interacting with constant magnetic field. The effective potential is evaluated and the correspondent phase structure is given. Some discussion and outlook are presented in

\footnotetext{
${ }^{\S}$ For the works where four-fermion models have been discussed in the presence of constant electromagnetic fields, see 10, 11, 12
} 
the last section.

\section{Scalar and spinor Green functions in the magnetic field in curved spacetime}

One of the fundamental objects in the field theory is a Green function. It is a basic quantity for a number of calculations of quantum effects. Here we calculate the scalar and spinor Green functions in the magnetic field in curved spacetime to study the phase structure of the NJL and SUSY NJL model in the external gravitational and constant magnetic fields.

\subsection{Green function for a scalar field}

First we study GF for the scalar field $G(x, y ; m)$ in an external electromagnetic and gravitational field. We assume that the spacetime curves slowly and neglect the terms involving the metric derivatives higher than third (weak curvature approximation). However we treat the external magnetic field exactly. The GF for the scalar field $G(x, y ; m)$ satisfies the Klein-Gordon equation :

$$
\left(i D^{\mu} i D_{\mu}-m^{2}-\xi R\right) G(x, y ; m)=\frac{1}{\sqrt{-g}} \delta^{D}(x-y)
$$

where $\xi$ represents the non-minimal coupling constant with external gravitational field(see [24]), $m$ is the scalar field mass and $D^{\mu}=\nabla^{\mu}-i e A^{\mu}$. In the present paper the vector potential of the external electromagnetic field is chosen in the form

$$
A_{\mu}(x)=-\frac{1}{2} F_{\mu \nu} x^{\nu}
$$

where $F_{\mu \nu}$ is constant matrix of electromagnetic field strength tensor. The covariant derivative for a scalar field is only an ordinary derivative $\nabla^{\mu}=\partial^{\mu}$. For a vector field $v^{\mu}$ the covariant derivative is rewritten as

$$
\begin{aligned}
\nabla_{\mu} v^{\mu} & =\partial_{\mu} v^{\mu}+\Gamma_{\nu \mu}^{\nu} v^{\mu} \\
& =\frac{1}{\sqrt{-g}} \partial_{\mu} \sqrt{-g} v^{\mu} .
\end{aligned}
$$

Thus the first term of the left hand side in Eq.(1) is rewritten as

$$
\begin{aligned}
\sqrt{-g} D^{\mu} D_{\mu} G(x, y ; m) & =\sqrt{-g}\left(\nabla^{\mu}-i e A^{\mu}\right)\left(\nabla_{\mu}-i e A_{\mu}\right) G(x, y ; m) \\
& =\left(\partial_{\mu}-i e A_{\mu}\right) \sqrt{-g} g^{\mu \nu}\left(\partial_{\nu}-i e A_{\nu}\right) G(x, y ; m) .
\end{aligned}
$$

We want to expand Eq.(1) around $R=0$. For this purpose we introduce the Riemann normal coordinates [16]. In the Riemann normal coordinates framework the metric tensor 
is expanded as

$$
\begin{aligned}
g_{\mu \nu}(y) & =\eta_{\mu \nu}+\frac{1}{3} R_{\mu \rho \sigma \nu}^{(0)}(y-x)^{\rho}(y-x)^{\sigma} \\
g(y) & =-1-\frac{1}{3} R_{\mu \nu}^{(0)}(y-x)^{\mu}(y-x)^{\nu}
\end{aligned}
$$

where the suffix (0) for $R_{\mu \rho \sigma \nu}^{(0)}$ and $R_{\mu \nu}^{(0)}$ designates the curvature tensor at the origin $x$. Substituting Eq.(司) into Eq.(四) we obtain

$$
\begin{aligned}
& \sqrt{-g} D^{\mu} D_{\mu} G(x, y ; m)=\left\{\left[\eta^{\mu \nu}+\frac{1}{6} R^{(0)}{ }_{\alpha \beta}(y-x)^{\alpha}(y-x)^{\beta} \eta^{\mu \nu}\right.\right.
\end{aligned}
$$

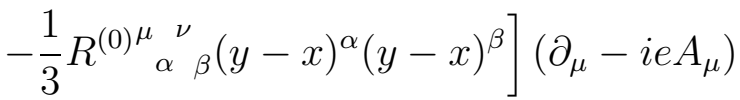

$$
\begin{aligned}
& \left.-\frac{2}{3} R_{\alpha}^{(0)^{\nu}}(y-x)^{\alpha}\right\}\left(\partial_{\nu}-i e A_{\nu}\right) G(x, y ; m) \text {, }
\end{aligned}
$$

where we keep only terms independent of curvature or linear in curvature. We expand the $\mathrm{GF}, G(x, y ; m)$, as

$$
G(x, y ; m)=G^{(0)}(x, y ; m)+G^{(1)}(x, y ; m)+\mathcal{O}\left(R^{2}\right),
$$

where $G^{(0)}$ and $G^{(1)}$ represent the terms independent of $R$ and the terms linear in $R$ respectively.

Substituting Eqs.(6) and (7) into (1) we can perturbatively solve the Klein-Gordon equation (1) about the spacetime curvature $R$. The piece independent of curvature in Eq.(11) is given by

$$
\left[\eta^{\mu \nu}\left(\partial_{\mu}-i e A_{\mu}\right)\left(\partial_{\nu}-i e A_{\nu}\right)+m^{2}\right] G^{(0)}(x, y ; m)=-\delta^{D}(x-y)
$$

Thus $G^{(0)}(x, y ; m)$ satisfies the Klein-Gordon equation in flat spacetime. We will give the explicit expression for $G^{(0)}(x, y ; m)$ below. The piece linear in $R$ in Eq.(1) becomes

$$
\begin{aligned}
& \left\{\left[\frac{1}{6} R^{(0)}{ }_{\alpha \beta}(y-x)^{\alpha}(y-x)^{\beta} \eta^{\mu \nu}\right.\right.
\end{aligned}
$$

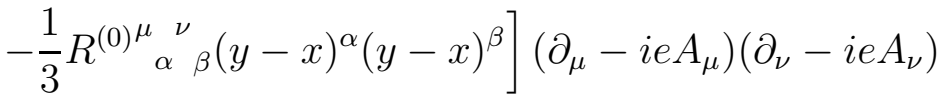

$$
\begin{aligned}
& \left.-\frac{2}{3} R^{(0)^{\nu}}{ }_{\alpha}(y-x)^{\alpha}\left(\partial_{\nu}-i e A_{\nu}\right)+\xi R\right\} G^{(0)}(x, y ; m) \\
& +\left[\eta^{\mu \nu}\left(\partial_{\mu}-i e A_{\mu}\right)\left(\partial_{\nu}-i e A_{\nu}\right)+m^{2}\right] G^{(1)}(x, y ; m)=0 \text {. }
\end{aligned}
$$

Hence the piece $G^{(1)}$ involving the terms linear in $R$ is expressed with the help of the GF $G^{(0)}(x, y ; m)$ in flat spacetime (for more details of local momentum representation, see [5, 15, 16, 17). 
It is more convenient to introduce the new variable defined by

$$
G(x, y ; m) \equiv \Phi(x, y) \tilde{G}(x-y ; m)
$$

where $\Phi(x, y)$ satisfies

$$
\left(\partial_{\mu}-i e A_{\mu}\right) \Phi(x, y)=0
$$

Inserting Eq.(10) into Eq.(9) we obtain

$$
\begin{aligned}
& {\left[\frac{1}{6} R^{(0)}{ }_{\alpha \beta}(y-x)^{\alpha}(y-x)^{\beta} \partial^{\mu} \partial_{\mu}\right.}
\end{aligned}
$$

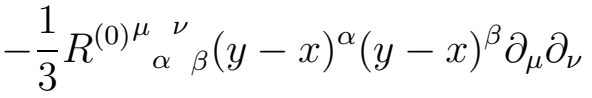

$$
\begin{aligned}
& \left.-\frac{2}{3} R^{(0)^{\nu}}{ }_{\alpha}(y-x)^{\alpha} \partial_{\nu}+\xi R^{(0)}\right] \tilde{G}^{(0)}(y-x ; m) \\
& +\left(\partial_{\mu} \partial_{\mu}+m^{2}\right) \tilde{G}^{(1)}(x-y ; m)=0 .
\end{aligned}
$$

$A_{\mu}$-dependence disappears in the relationship between $\tilde{G}^{(0)}(y-x ; m)$ and $\tilde{G}^{(1)}(x-y ; m)$. Therefore the GF $\tilde{G}^{(1)}(x-y ; m)$ is given by

$$
\begin{aligned}
& \tilde{G}^{(1)}(x-y ; m)=\int d^{D} z G_{00}(x-z ; m) \\
& \times\left[\frac{1}{6} R_{\alpha \beta}^{(0)}(z-y)^{\alpha}(z-y)^{\beta} \partial^{\mu} \partial_{\mu}\right.
\end{aligned}
$$

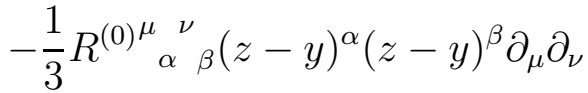

$$
\begin{aligned}
& \left.-\frac{2}{3} R^{(0)^{\nu}}{ }_{\alpha}(z-y)^{\alpha} \partial_{\nu}+\xi R^{(0)}\right] \tilde{G}^{(0)}(z-y ; m) \text {, }
\end{aligned}
$$

where the function $G_{00}(x-y ; m)$ satisfies

$$
\left(\partial^{\mu} \partial_{\mu}+m^{2}\right) G_{00}(x-y ; m)=-\delta^{D}(x-y) .
$$

The linear curvature correction terms of the scalar GF are expressed by the GF in flat spacetime.

In the constant curvature spacetime,

$$
R_{\mu \nu \rho \sigma}=\frac{R}{D(D-1)}\left(\eta_{\mu \rho} \eta_{\nu \sigma}-\eta_{\mu \sigma} \eta_{\nu \rho}\right),
$$

the equation (13) simplifies to

$$
\begin{aligned}
& \tilde{G}^{(1)}(x-y ; m) \\
& =\frac{R}{D(D-1)} \int d^{D} z G_{00}(x-z ; m) \\
& \times\left[\frac{D-3}{6} \eta_{\alpha \beta}(z-y)^{\alpha}(z-y)^{\beta} \partial^{\mu} \partial_{\mu}+\frac{1}{3}(z-y)^{\mu}(z-y)^{\nu} \partial_{\mu} \partial_{\nu}\right. \\
& \left.-\frac{2}{3}(D-1)(z-y)^{\mu} \partial_{\mu}+D(D-1) \xi\right] \tilde{G}^{(0)}(z-y ; m) .
\end{aligned}
$$


As is shown in Eq.(\$), $G^{(0)}(x, y ; m)$ satisfies the Klein-Gordon equation in flat spacetime. According to the Schwinger proper-time method the Klein-Gordon equation is exactly solvable in the constant electromagnetic field $[18,19]$. To calculate $G^{(0)}(x, y ; m)$ we introduce the proper-time Hamiltonian which is defined by

$$
H\left(x^{\mu}, i \partial_{\mu}\right) G^{(0)}(x, y ; m) \equiv\left[\left(i \partial^{\mu}+e A^{\mu}\right)\left(i \partial_{\mu}+e A_{\mu}\right)-m^{2}\right] G^{(0)}(x, y ; m) .
$$

For this proper-time Hamiltonian the time evolution operator $U(x, y ; s)$ is defined by

$$
i \frac{\partial}{\partial s} U(x, y ; s)=H\left(x, i \partial_{\mu}\right) U(x, y ; s)
$$

with the boundary conditions

$$
\left\{\begin{array}{l}
\lim _{s \rightarrow 0} U(x, y ; s)=\delta^{D}(x-y) \\
\lim _{s \rightarrow-\infty} U(x, y ; s)=0
\end{array}\right.
$$

Comparing Eq.(19) with Eq.(18) we find

$$
G^{(0)}(x, y ; m)=-i \int_{-\infty}^{0} d s U(x, y ; s)
$$

The time evolution operator $U(x, y ; s)$ is obtained by solving the equation of motion for $x^{\mu}$ and $\pi^{\mu}=i \partial^{\mu}-e A^{\mu}$. For a constant electromagnetic field, $x^{\mu}(s)=U^{\dagger}(s) x^{\mu} U(s)$ and $\pi^{\mu}(s)=U^{\dagger}(s) \pi^{\mu} U(s)$ satisfy the following equations :

$$
\begin{gathered}
\frac{d x^{\mu}(s)}{d s}=i\left[H, x^{\mu}\right]=-2 \pi^{\mu} . \\
\frac{d \pi_{\mu}(s)}{d s}=i\left[H, \pi_{\mu}\right]=2 e F_{\mu \nu} \pi^{\nu} .
\end{gathered}
$$

These differential equations can be easily solved,

$$
\begin{gathered}
\pi(s)=e^{2 e F s} \pi(0), \\
x(s)-x(0)=-\frac{e^{2 e F s}-1}{e F} \pi(0),
\end{gathered}
$$

where we use the matrix notation. Substituting Eqs.(24) and (25) into Eq.(19) we obtain the differential equation for the time evolution operator $U(x, y ; s)$,

$$
\begin{aligned}
& i \frac{\partial}{\partial s} U(x, y ; s)=\left(\pi^{2}(s)-m^{2}\right) U(x, y ; s) \\
& =\left\{(x-y) K(x-y)-\frac{i}{2} \operatorname{tr}[e F \operatorname{coth}(e F s)]-m^{2}\right\} U(x, y ; s),
\end{aligned}
$$

I For other types of exact solutions of the Klein-Gordon equation in the external electromagnetic field, see [20. 
where $K$ is defined by

$$
K \equiv \frac{1}{4} e^{2} F^{2}[\sinh (e F s)]^{-2} .
$$

After the integration over $s$ in Eq.(26) the time evolution operator is found to be :

$$
\begin{aligned}
U(x, y ; s)= & -\frac{i}{(4 \pi)^{D / 2}} \Phi(x, y) s^{-D / 2} \exp \left\{-\frac{1}{2} \operatorname{tr} \ln \left[\frac{\sinh (e F s)}{e F s}\right]\right\} \\
& \times \exp \left[\frac{i}{4}(x-y) e F \operatorname{coth}(e F s)(x-y)+i m^{2} s\right] .
\end{aligned}
$$

Substituting this time evolution operator (28) to Eq.(21) we find the GF, $G^{(0)}(x, y ; m)$,

$$
\begin{aligned}
& G^{(0)}(x, y ; m)=-i \int_{-\infty}^{0} d s U(x, y ; s) \\
& =-\frac{1}{(4 \pi)^{D / 2}} \Phi(x, y) \int_{-\infty}^{0} d s s^{-D / 2} \exp \left[-\frac{1}{2} \operatorname{tr} \ln \left(\frac{\sinh (e F s)}{e F s}\right)\right] \\
& \times \exp \left[\frac{i}{4}(x-y) e F \operatorname{coth}(e F s)(x-y)+i m^{2} s\right] .
\end{aligned}
$$

Taking the limit $F \rightarrow 0$ and changing the variable $s \rightarrow-s$ in Eq.(29) we get the GF, $G_{00}(x-y ; m)$,

$$
\begin{aligned}
& G_{00}(x-y ; m)=-\int_{0}^{\infty} d s \frac{1}{(4 \pi s)^{D / 2}} \\
& \times \exp \left[-i \frac{\pi}{4} D-\frac{i}{4 s}(x-y)^{\mu}(x-y)_{\mu}-i m^{2} s\right],
\end{aligned}
$$

where the phase factor is determined to satisfy the boundary condition (20).

In the present paper we consider the constant magnetic field along the $z$-axis. For this constant magnetic field, $F_{12}=-F_{21}=B$, the GF, $\tilde{G}^{(0)}(x-y ; m)$, reduces to

$$
\begin{aligned}
& \tilde{G}^{(0)}(x-y ; m)=-\int_{0}^{\infty} d s \frac{1}{(4 \pi s)^{D / 2}} \\
& \times \frac{e B s}{\sin (e B s)} \exp \left[-i \frac{\pi}{4} D-\frac{i}{4 s}(x-y)^{\mu} C_{\mu \nu}(x-y)^{\nu}-i m^{2} s\right],
\end{aligned}
$$

where

$$
C_{\mu \nu}=\eta_{\mu \nu}+F_{\mu}{ }^{\lambda} F_{\lambda \nu} \frac{1-e B s \cot (e B s)}{B^{2}} .
$$

Inserting the Eqs.(30) and (31) into (17) we obtain the GF linear in $R$

$$
\begin{aligned}
& \tilde{G}^{(1)}(x-y ; m)=\frac{R}{D(D-1)} \int d^{D} z \int_{0}^{\infty} \frac{d t}{(4 \pi t)^{D / 2}} \int_{0}^{\infty} \frac{d s}{(4 \pi s)^{D / 2}} \\
& \times \exp \left[-i \frac{\pi}{4} D-\frac{i}{4 t}(x-z)^{\mu}(x-z)_{\mu}-i m^{2} t\right]
\end{aligned}
$$




$$
\begin{aligned}
& \times\left[\frac{D-3}{6} \eta_{\alpha \beta}(z-y)^{\alpha}(z-y)^{\beta} \partial^{\mu} \partial_{\mu}\right. \\
& \left.+\frac{1}{3}(z-y)^{\mu}(z-y)^{\nu} \partial_{\mu} \partial_{\nu}-\frac{2}{3}(D-1)(z-y)^{\mu} \partial_{\mu}+D(D-1) \xi\right] \\
& \times \frac{e B s}{\sin (e B s)} \exp \left[-i \frac{\pi}{4} D-\frac{i}{4 s}(z-y)^{\mu} C_{\mu \nu}(z-y)^{\nu}-i m^{2} s\right]
\end{aligned}
$$

Thus, we obtain the explicit expression of the scalar GF in the external gravitational and magnetic field.

We need only the coincidence limit $x \rightarrow y$ of the GF to calculate the effective potential of the models considered in the present paper. After the Wick rotation $s \rightarrow-i s, t \rightarrow$ $-i t, z^{0} \rightarrow-i z^{0}$ and the integration over $z \operatorname{Sp} G(x, x ; m)$ simplifies to

$$
\begin{aligned}
& \operatorname{SpG}(0)(x, x ; m)=\frac{-i}{(4 \pi)^{D / 2}} \int_{0}^{\infty} d s s^{-D / 2} \frac{e B s}{\sinh (e B s)} \exp \left(-m^{2} s\right) \\
& \operatorname{Sp} G^{(1)}(x, x ; m)=\frac{i}{(4 \pi)^{D / 2}} \frac{R}{D(D-1)} \int_{0}^{\infty} \int_{0}^{\infty} d t d s \exp \left[-m^{2}(s+t)\right] \\
& \times \frac{(s+t)^{(2-D) / 2}}{1+e B t \operatorname{coth}(e B s)} \frac{e B}{\sinh (e B s)} \\
& \times\left\{\left[-\frac{D-3}{6}(D-2+2 e B s \operatorname{coth}(e B s))+\frac{-2 D+1}{3}\right] \frac{(D-2) t}{s+t}\right. \\
& +\left[-\frac{D-3}{6 s}(D-2+2 e B s \operatorname{coth}(e B s))+\frac{-2 D+1}{3} e B \operatorname{coth}(e B s)\right] \\
& \times \frac{2 t}{1+e B t \operatorname{coth}(e B s)} \\
& +\frac{2(D-1)}{3}\left[\frac{D(D-2)}{4}\left(\frac{t}{s+t}\right)^{2}+2\left(\frac{e B t \operatorname{coth}(e B s)}{1+e B t \operatorname{coth}(e B s)}\right)^{2}\right] \\
& +\frac{1}{3 s}\left[(D-3)\left((e B s)^{2} \operatorname{coth}^{2}(e B s)+1\right)+4 e B s \operatorname{coth}(e B s)\right] \\
& \left.\times \frac{(D-2) t}{s+t} \frac{t}{1+e B t \operatorname{coth}(e B s)}+D(D-1) \xi\right\} .
\end{aligned}
$$

Eqs.(34) and (35) correspond to the the vacuum self-energy of the free scalar field with mass $m$ at the one loop level.

\subsection{Green function for a spinor field}

Next we construct the spinor GF in an external electromagnetic and gravitational field. Let us write the GF, which obeys the Dirac equation:

$$
\left(i \gamma^{\mu} D_{\mu}-m\right) S(x, y ; m)=\frac{1}{\sqrt{-g}} \delta^{D}(x-y),
$$


where the covariant derivative $D_{\mu}$ includes the electromagnetic potential $A_{\mu}$ :

$$
D_{\mu}=\partial_{\mu}-i e A_{\mu}+\frac{1}{2} \omega^{a b}{ }_{\mu} \sigma_{a b} .
$$

The local Dirac matrices $\gamma_{\mu}(x)$ are expressed through the usual flat ones $\gamma_{a}$ and tetrads $e_{\mu}^{a}$ :

$$
\begin{aligned}
& \gamma^{\mu}(x)=\gamma^{a} e_{a}^{\mu}(x), \\
& \sigma_{a b}=\frac{1}{4}\left[\gamma_{a}, \gamma_{b}\right] .
\end{aligned}
$$

The spin-connection has the form :

$$
\begin{aligned}
\omega^{a b}{ }_{\mu} & =\frac{1}{2} e^{a \nu}\left(\partial_{\mu} e_{\nu}^{b}-\partial_{\nu} e_{\mu}^{b}\right)+\frac{1}{4} e^{a \nu} e^{b \rho} e_{c \mu}\left(\partial_{\rho} e_{\nu}^{c}-\partial_{\nu} e_{\rho}^{a}\right) \\
& -\frac{1}{2} e^{b \nu}\left(\partial_{\mu} e_{\nu}^{a}-\partial_{\nu} e_{\mu}^{a}\right)-\frac{1}{4} e^{b \nu} e^{a \rho} e_{c \mu}\left(\partial_{\rho} e_{\nu}^{c}-\partial_{\nu} e_{\rho}^{c}\right) .
\end{aligned}
$$

Dimensions of the spinor representation are supposed to be four. Greek and Latin indices correspond to the curved and flat tangent spacetimes.

To calculate the linear curvature corrections the local momentum expansion formalism as in the previous subsection is the most convenient one [5, 15, 17]. In the Riemann normal coordinates framework the tetrads $e^{\mu}{ }_{a}(x)$ and the spin connection $\omega^{a b}{ }_{\mu} \sigma_{a b}$ are expanded as

$$
\begin{aligned}
e^{\mu}{ }_{a}(y) & =\delta^{\mu}{ }_{a}+\frac{1}{6} R^{(0) \mu}{ }_{\rho \sigma a}(y-x)^{\rho}(y-x)^{\sigma}, \\
\omega^{a b}{ }_{\mu} \sigma_{a b} & =\frac{1}{2} R^{(0) a b}{ }_{\mu \lambda}(y-x)^{\lambda} \sigma_{a b} .
\end{aligned}
$$

Substituting (39) and (40) into the (37), we obtain the following equation for the GF :

$$
\begin{aligned}
& {\left[i \gamma^{a}\left(\delta^{\mu}{ }_{a}+\frac{1}{6} R_{\rho \sigma a}^{(0) \mu}(y-x)^{\rho}(y-x)^{\sigma}\right)\left(\partial_{\mu}+\frac{1}{4} R_{b c \mu \lambda}^{(0)}(y-x)^{\lambda} \sigma^{b c}-i e A_{\mu}\right)\right.} \\
& -m] S(x, y ; m)=\delta^{D}(x-y) .
\end{aligned}
$$

Fulfilling the expansion on the spacetime curvature degrees

$$
S=S^{(0)}+S^{(1)}+\cdots,
$$

where $S^{(0)}$ is the GF in the flat spacetime, $S^{(1)} \sim \mathcal{O}(R)$ and so on, we receive the iterative sequence of equations:

$$
\begin{aligned}
& {[i \not \partial+e \not A(x)-m] S^{(0)}(x, y ; m)=\delta^{D}(x-y)} \\
& \quad\left[(i \not \partial+e \not A(x)-m) S^{(1)}(x, y ; m)\right. \\
& \quad+\frac{i}{6} \gamma^{a} R_{\rho \sigma a}^{(0) \mu}(y-x)^{\rho}(y-x)^{\sigma}\left(\partial_{\mu}-i e A_{\mu}(x)\right) \\
& \left.\quad+\frac{i}{4} \gamma^{a} R_{b c a \lambda}^{(0)}(y-x)^{\lambda} \sigma^{b c}\right] S^{(0)}(x, y ; m)=0 .
\end{aligned}
$$


Here and below we can forget about the difference between the two kinds of indices (Greek and Latin) because it lies beyond of linear curvature approximation.

We assume that just as in the flat spacetime GF has the form [18]:

$$
S(x, y ; m)=\Phi(x, y) \tilde{S}(x-y ; m),
$$

where the function $\Phi(x, y)$ is introduced in Eq.([1]). Then, we can find the equation which determines the $\tilde{S}^{(1)}(x-y ; m)$ function, excluding the evident dependence on $A_{\mu}(x)$ :

$$
\begin{aligned}
& (i \not \partial-m) \tilde{S}^{(1)}(x-y ; m) \\
& =-\frac{i}{6} \gamma^{a} R_{\rho \sigma a}^{(0) \mu}(y-x)^{\rho}(y-x)^{\sigma} \partial_{\mu} \tilde{S}^{(0)}(x-y ; m) \\
& -\frac{i}{4} \gamma^{a} \sigma^{b c} R_{b c a \lambda}^{(0)}(y-x)^{\lambda} \tilde{S}^{(0)}(x-y ; m) .
\end{aligned}
$$

The flat spacetime GF in the external electromagnetic field is supposed to be known [11, 12, 18]. Denoting as $S_{00}(x-y ; m)$ the GF, satisfying the equation

$$
(i \not \partial-m) S_{00}(x-y ; m)=\delta^{D}(x-y),
$$

we obtain

$$
\begin{aligned}
& \int d^{D} z S_{00}^{-1}(x-z ; m) \tilde{S}^{(1)}(z-y ; m) \\
& =-\frac{i}{6} \gamma^{a} R_{\rho \sigma a}^{(0) \mu}(x-y)^{\rho}(x-y)^{\sigma} \partial_{\mu} \tilde{S}^{(0)}(x-y ; m) \\
& -\frac{i}{4} \gamma^{a} \sigma^{b c} R_{b c a \lambda}^{(0)}(x-y)^{\lambda} \tilde{S}^{(0)}(x-y ; m),
\end{aligned}
$$

or, finally,

$$
\begin{aligned}
& \tilde{S}^{(0)}(x-y ; m)=\int d^{D} z S_{00}(x-z ; m) \\
& \times\left[-\frac{i}{6} \gamma^{a} R_{\rho \sigma a}^{(0) \mu}(z-y)^{\rho}(z-y)^{\sigma} \partial_{\mu} \tilde{S}^{(0)}(z-y ; m)\right. \\
& \left.-\frac{i}{4} \gamma^{a} \sigma^{b c} R_{b c a \lambda}^{(0)}(z-y)^{\lambda}\right] \tilde{S}_{0}(z-y ; m) .
\end{aligned}
$$

However, we need only the coincidence limit $x \rightarrow y$ to calculate the effective potential. It provides us the opportunity to simplify (49) especially for the constant curvature spacetimes (15). Thus the expression for the GF, $\tilde{S}^{(1)}$, in the spacetime with an arbitrary dimension $D$ is the following:

$$
\begin{aligned}
\tilde{S}^{(1)}(0 ; m)= & -\frac{i R}{12 D(D-1)} \int d^{D} z S_{00}(-z ; m)\left[2 \not z z^{\mu} \partial_{\mu} \tilde{S}^{(0)}(z ; m)\right. \\
& \left.-2 z^{2} \gamma^{\mu} \partial_{\mu} \tilde{S}^{(0)}(z ; m)+3(D-1) \not z \tilde{S}^{(0)}(z ; m)\right] .
\end{aligned}
$$


Now we begin the calculation of the Green function for the $D=4$ Gross-Neveu model in the external magnetic field. According to the Schwinger proper-time method as in the previous subsection the flat spacetime GF is found to be [11, 18]:

$$
\begin{aligned}
& \tilde{S}^{(0)}(z ; m)=-i \int_{0}^{\infty} \frac{d s}{16(\pi s)^{2}} \exp \left[-i\left(\frac{\pi}{2}+s m^{2}\right)\right] \exp \left(-\frac{i}{4 s} z_{\mu} C^{\mu \nu} z_{\nu}\right) \\
& \times\left(m+\frac{1}{2 s} \gamma^{\mu} C_{\mu \nu} z^{\nu}-\frac{e}{2} \gamma^{\mu} F_{\mu \nu} z^{\nu}\right)\left[(e B s) \cot (e B s)-\frac{e s}{2} \gamma^{\mu} \gamma^{\nu} F_{\mu \nu}\right]
\end{aligned}
$$

where $C_{\mu \nu}$ is defined in Eq.(32). For $S_{00}(-z ; m)$ we have directly from (51):

$$
S_{00}(-z ; m)=-i \int_{0}^{\infty} \frac{d t}{16(\pi t)^{2}} \exp \left[-i\left(\frac{\pi}{2}+m^{2} t+\frac{z^{2}}{4 t}\right)\right]\left(m-\frac{\not z}{2 t}\right) .
$$

Substituting (52) in (50) and calculating the trace over the spinor indices, we have :

$$
\begin{aligned}
S p \tilde{S}^{(1)}(0 ; m)= & \frac{-i R m}{36} \int \frac{d^{4} z d t d s}{\left(16 \pi^{2} t s\right)^{2}} \\
& \times \exp \left\{-i\left[(t+s) m^{2}+\frac{t+s}{4 t s} z_{\|}^{2}-z_{\perp}^{2} \frac{1+e B t \cot (e B s)}{4 t}\right]\right\} \\
& \times\left\{e B s \operatorname { c o t } ( e B s ) \left[z_{\|}^{2}\left(-\frac{9}{2 t}+\frac{7-4 e B s \cot (e B s)}{2 s}\right)\right.\right. \\
& +z_{\perp}^{2}\left(\frac{9}{2 t}+\frac{4-7 e B s \cot (e B s)}{2 s}\right) \\
& \left.-\frac{i}{2 s^{2}} z_{\|}^{2} z_{\perp}^{2}(1-e B s \cot (e B s))^{2}\right]-e^{2} B^{2}\left[s\left(2 z_{\|}^{2}+\frac{7}{2} z_{\perp}^{2}\right)\right. \\
& \left.\left.+\frac{i}{2} z_{\|}^{2} z_{\perp}^{2}(e B s \cot (e B s)-1)\right]\right\}
\end{aligned}
$$

where

$$
z_{\perp}^{2}=z_{1}^{2}+z_{2}^{2}, z_{\|}^{2}=z_{0}^{2}-z_{3}^{2} .
$$

After the Wick rotation and integration over $z$, one gets:

$$
\begin{aligned}
S p \tilde{S}^{(1)}(0 ; m)= & \frac{i R m}{96 \pi^{2}} \int \frac{d t d s}{(t+s)^{2}(1+e B t \operatorname{coth}(e B s))^{2}} \exp \left[-(t+s) m^{2}\right] \\
& \times[e B t(e B t+e B s)+2(e B t+3 e B s) \operatorname{coth}(e B s) \\
& \left.+2 e B t(e B s-e B t) \operatorname{coth}^{2}(e B s)\right] .
\end{aligned}
$$

The same program can be done for the 3D case. The GF in the flat space-time with the external constant magnetic field has the following form [11, 12]:

$$
\tilde{S}^{(0)}(z ; m)=-i \int_{0}^{\infty} \frac{d s}{8(\pi s)^{3 / 2}} e^{-i\left(\pi / 4+s m^{2}\right)} \exp \left(-\frac{i}{4 s} z_{\mu} C^{\mu \nu} z_{\nu}\right)
$$




$$
\begin{aligned}
& \times\left(m+\frac{1}{2 s} \gamma^{\mu} C_{\mu \nu} z^{n u}-\frac{e}{2} \gamma^{\mu} F_{\mu \nu} z^{\nu}\right) \\
& \times\left[e B s \cot (e B s)-\frac{e s}{2} \gamma^{\mu} \gamma^{\nu} F_{\mu \nu}\right]
\end{aligned}
$$

For $S_{00}(-z ; m)$ we have directly from $(56)$ :

$$
S_{00}(-z ; m)=-i \int_{0}^{\infty} \frac{d t}{8(\pi t)^{3 / 2}} \exp \left[-i\left(\frac{\pi}{4}+m^{2} t+\frac{z^{2}}{4 t}\right)\right]\left(m-\frac{\not z}{2 t}\right) .
$$

Substituting (56),(57) in (50), we have:

$$
\begin{aligned}
S p \tilde{S}^{(1)}(0 ; m)= & \frac{R m}{1152 \pi^{3}} \int \frac{d^{3} z d t d s}{(t s)^{3 / 2}} \exp \left\{-i\left[(t+s) m^{2}+\frac{t+s}{4 t s} z_{0}^{2}\right.\right. \\
& \left.\left.-z_{\perp}^{2} \frac{1+e B t \cot (e B s)}{4 t}\right]\right\} \\
& \times\left\{e B s \operatorname { c o t } ( e B s ) \left[z_{0}^{2}\left(-\frac{3}{t}+\frac{3-2 e B s \cot (e B s)}{s}\right)\right.\right. \\
& +z_{\perp}^{2}\left(\frac{3}{t}+\frac{1-2 e B s \cot (e B s)}{s}\right) \\
& \left.-\frac{i}{2 s^{2}} z_{0}^{2} z_{\perp}^{2}(1-e B s \cot (e B s))^{2}\right] \\
& +e^{2} B^{2}\left[-2 s\left(z_{0}^{2}+z_{\perp}^{2}\right)\right. \\
& \left.\left.+\frac{i}{2} z_{0}^{2} z_{\perp}^{2}(1-e B s \cot (e B s))\right]\right\}
\end{aligned}
$$

After the Wick rotation and integration over $z$, one gets:

$$
\begin{aligned}
& S p \tilde{S}^{(1)}(0 ; m)=\frac{i R m}{72 \pi^{3 / 2}} \int \frac{d t d s}{(t+s)^{3 / 2}(1+e B t \operatorname{coth}(e B s))^{2}} \exp \left[-(t+s) m^{2}\right] \\
& \times[2 e B t(e B t+e B s)+(9 e B s+5 e B t) \operatorname{coth}(e B s) \\
& \left.+e B t(e B s-3 e B t) \operatorname{coth}^{2}(e B s)\right] .
\end{aligned}
$$

The proper-time integrations remain in our final expressions of the scalar and spinor Green functions in an external magnetic and gravitational field. All remained integrands are exponentially suppressed at the limit $s \rightarrow \infty$ and/or $t \rightarrow \infty$. There are divergences at $s \rightarrow 0$ and/or $t \rightarrow 0$ for $D \geq 2$.

\section{$3 \quad$ NJL model}

Let us discuss now the NJL model [1] in an external magnetic and gravitational field (for a review, see [5]). The NJL model is one of the simplest models where dynamical 
symmetry breaking is possible. It is well-know that the chiral symmetry of the model is dynamically broken down for a sufficiently large coupling constant. In the present paper we want to know the effect of an external electromagnetic and gravitational field to the dynamical symmetry breaking.

The NJL model is defined by the action which is given by

$$
S=\int d^{D} x \sqrt{-g}\left\{i \bar{\psi} \gamma^{\mu}(x) D_{\mu} \psi+\frac{\lambda}{2 N}\left[(\bar{\psi} \psi)^{2}+\left(\bar{\psi} i \gamma_{5} \psi\right)^{2}\right]\right\}
$$

where $N$ is the number of the fermions. This action has the chiral $U(1)$ symmetry. Introducing the auxiliary fields

$$
\sigma=-\frac{\lambda}{N}(\bar{\psi} \psi), \pi=-\frac{\lambda}{N} \bar{\psi} i \gamma_{5} \psi
$$

we can rewrite the action (60) as:

$$
S=\int d^{D} x \sqrt{-g}\left[i \bar{\psi} \gamma^{\mu} D_{\mu} \psi-\frac{N}{2 \lambda}\left(\sigma^{2}+\pi^{2}\right)-\bar{\psi}\left(\sigma+i \pi \gamma_{5}\right) \psi\right] .
$$

In the leading order of the $1 / N$ expansion the effective action is given by

$$
\frac{1}{N} \Gamma_{e f f}(\sigma, \pi)=-\int d^{D} x \sqrt{-g} \frac{\sigma^{2}+\pi^{2}}{2 \lambda}-i \ln \operatorname{det}\left[i \gamma^{\mu}(x) D_{\mu}-\left(\sigma+i \gamma_{5} \pi\right)\right] .
$$

We can put now $\pi=0$ because the final expression would depend on the combination $\sigma^{2}+\pi^{2}$ only. In this case the effective action of the NJL model has the same form as the one of the Gross-Neveu model [2]. Note that Eq.(63) represents the particular example of the effective action for composite fields [21].

Defining the effective potential as $V_{\text {eff }}=-\Gamma_{\text {eff }} / N \int d^{D} x \sqrt{-g}$ for constant configurations of $\sigma$ and $\pi$, we obtain

$$
V_{e f f}(\sigma)=\frac{\sigma^{2}}{2 \lambda}+i S p \ln \left\langle x\left|\left[i \gamma^{\mu}(x) D_{\mu}-\sigma\right]\right| x\right\rangle .
$$

The second term of the right hand side in Eq.(64) is rewritten as

$$
i S p \ln \left\langle x\left|\left[i \gamma^{\mu}(x) D_{\mu}-\sigma\right]\right| x\right\rangle=-i S p \int^{\sigma} d m S(x, x ; m) .
$$

Thus we can express the effective potential of the NJL model by the spinor Green function $S(x, x, \sigma)$. Substituting the Green function evaluated in the previous section to Eq. (65) we obtain the effective potential in an external magnetic and gravitational field

In four-dimensional spacetime the linear curvature correction for the effective potential is given by

$$
\begin{aligned}
& V_{e f f}^{(1)}(\sigma)=-\frac{R}{192 \pi^{2}} \int_{1 / \Lambda^{2}}^{\infty} \int_{1 / \Lambda^{2}}^{\infty} \frac{d t d s}{(t+s)^{3}(1+e B t \operatorname{coth}(e B s))^{2}} \\
& \times \exp \left[-(t+s) \sigma^{2}\right][e B t(e B t+e B s)+2(e B t+3 e B s) \operatorname{coth}(e B s) \\
& \left.+2 e B t(e B s-e B t) \operatorname{coth}^{2}(e B s)\right],
\end{aligned}
$$


$V_{e f f}^{(0)}(\sigma) / \mu^{4}$

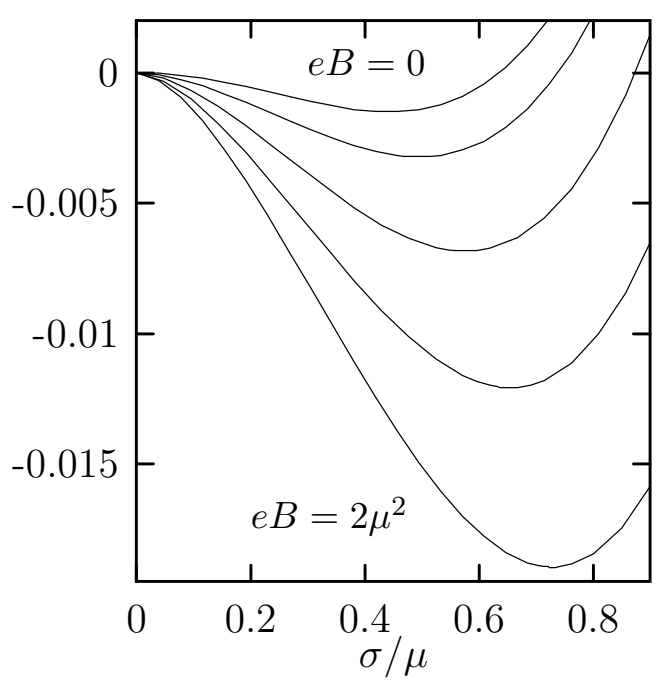

(a) $V_{\text {eff }}^{(0)}$ for $R=0$.
$V_{e f f}^{(1)}(\sigma) / \mu^{4}$

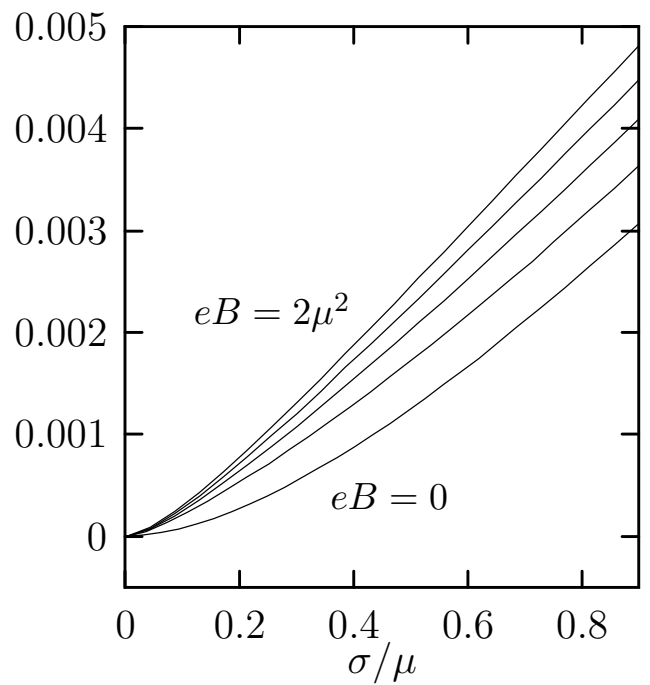

(b) $V_{e f f}^{(1)}$ for $R=0$.

Figure 1: The behavior of $V_{e f f}^{(0)}$ and $V_{e f f}^{(1)}$ are shown with the varying magnetic field $e B\left(=0, \mu^{2} / 2, \mu^{2}, 3 \mu^{2} / 2,2 \mu^{2}\right)$ for fixed $\lambda(=1 / 2.5)$ and fixed $\Lambda(=10 \mu)$ in four dimensional flat spacetime. $\mu$ is an arbitrary mass scale. We normalize that $V_{e f f}^{(0)}(0)=V_{\text {eff }}^{(1)}(0)=0$.

where we introduce the proper-time cut-off $\Lambda$. In four dimensions four-fermion models are not renormalizable. Here we define the finite theory by introducing the proper-time cut-off $\Lambda$. Taking into account the proper-time representation for $V_{\text {eff }}$ in four-dimensional flat spacetime [18], we obtain the effective potential with the linear curvature accuracy:

$$
\begin{aligned}
& V_{e f f}(\sigma)=\frac{\sigma^{2}}{2 \lambda}+\frac{1}{8 \pi^{2}} \int_{1 / \Lambda^{2}}^{\infty} \frac{d s}{s^{3}} \exp \left(-s \sigma^{2}\right) e B s \operatorname{coth}(e B s) \\
& -\frac{R}{192 \pi^{2}} \int_{1 / \Lambda^{2}}^{\infty} \int_{1 / \Lambda^{2}}^{\infty} \frac{d s d t}{(t+s)^{3}(1+e B t \operatorname{coth}(e B s))^{2}} \\
& \times \exp \left[-(t+s) \sigma^{2}\right][e B t(e B t+e B s)+2(e B t+3 e B s) \operatorname{coth}(e B s) \\
& \left.+2 e B t(e B s-e B t) \operatorname{coth}^{2}(e B s)\right] .
\end{aligned}
$$

It should be emphasised here that for $B=0$ we obtain just the same expression for $4 \mathrm{D}$ NJL model $V_{\text {eff }}$ in the proper-time representation as it has been found out in [14] (See also [5]).

For $D=3$ the linear curvature correction for the effective potential is given by

$$
\begin{aligned}
& V_{e f f}^{(1)}(\sigma)=-\frac{R}{144 \pi^{3 / 2}} \int_{1 / \Lambda^{2}}^{\infty} \int_{1 / \Lambda^{2}}^{\infty} \frac{d t d s}{(t+s)^{5 / 2}(1+e B t \operatorname{coth}(e B s))^{2}} \\
& \times \exp \left[-(t+s) \sigma^{2}\right][2 e B t(e B t+e B s)+(9 e B s+5 e B t) \operatorname{coth}(e B s) \\
& \left.+e B t(e B s-3 e B t) \operatorname{coth}^{2}(e B s)\right]
\end{aligned}
$$


$V_{\text {eff }}(\sigma) / \mu^{4}$

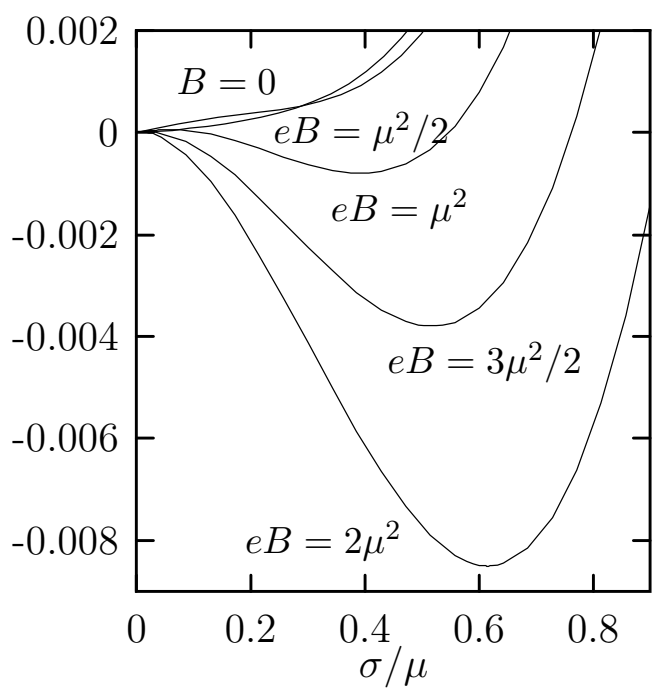

(a) $V_{\text {eff }}$ for $R=3 \mu^{2}$.
$V_{e f f}(\sigma) / \mu^{4}$

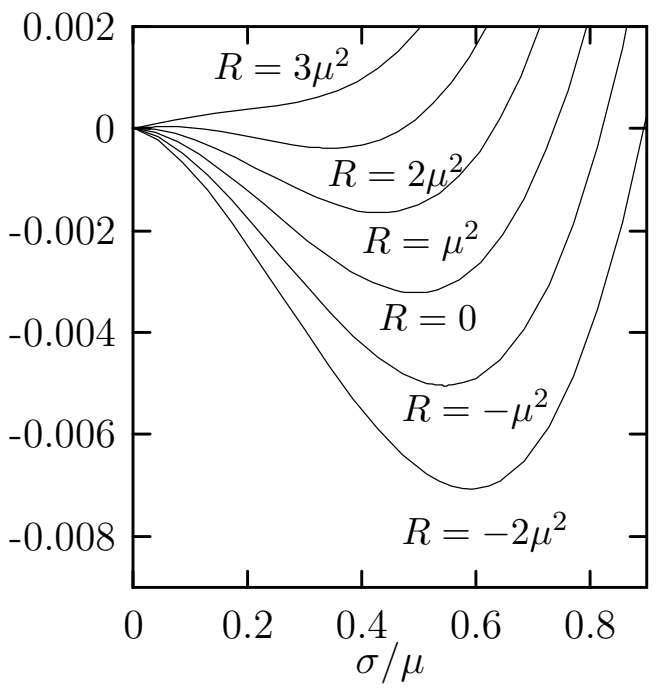

(b) $V_{\text {eff }}$ for $e B=\mu^{2} / 2$.

Figure 2: The behavior of the effective potential $V_{\text {eff }}$ is shown with the varying $B$ or $R$ for fixed $\lambda(=1 / 2.5)$ and fixed $\Lambda(=10 \mu)$ in four dimensions.

For $D=3$, four-fermion models are known to be renormalizable in the sense of the $1 / N$ expansion. Thus the cut-off dependence in the effective potential disappears after the usual renormalization procedure. Taking into account the proper-time representation for $V_{\text {eff }}$ in flat spacetime [11, 12], we can write the effective potential with the linear curvature accuracy:

$$
\begin{aligned}
& V_{e f f}(\sigma)=\frac{\sigma^{2}}{2 \lambda}+\frac{1}{4 \pi^{3 / 2}} \int_{1 / \Lambda^{2}}^{\infty} \frac{d s}{s^{5 / 2}} \exp \left(-s \sigma^{2}\right) e B s \operatorname{coth}(e B s) \\
& -\frac{R}{144 \pi^{3 / 2}} \int_{1 / \Lambda^{2}}^{\infty} \int_{1 / \Lambda^{2}}^{\infty} \frac{d s d t}{(t+s)^{5 / 2}(1+e B t \operatorname{coth}(e B s))^{2}} \\
& \times \exp \left[-(t+s) \sigma^{2}\right][2 e B t(e B t+e B s)+(9 e B s+5 e B t) \operatorname{coth}(e B s) \\
& \left.+e B t(e B s-3 e B t) \operatorname{coth}^{2}(e B s)\right] .
\end{aligned}
$$

The effective potential (69) gives again the correct expression for $V_{\text {eff }}$ of 3D NJL model in the proper-time representation with $B=0$ [13].

Using Eq. (67) we numerically calculate the effective potential for $D=4$ and show it in Figs.1 and 2. In drawing Fig. 1 and 2 the coupling constant $\lambda$ is kept in the region where the chiral symmetry is broken down for $R=B=0$. To see the curvature effect in the external constant magnetic field we divide the effective potential into the terms independent of the curvature and the terms linear in the curvature, $V_{e f f}=V_{e f f}^{(0)}+V_{e f f}^{(1)}$. In Fig. 1 typical behaviors of the effective potential $V_{e f f}^{(0)}$ and $V_{e f f}^{(1)}$ are given for fixed fourfermion coupling constant $\lambda$ and proper-time cut-off $\Lambda$. It is clearly seen in Figs. 1 and 
2.(a) that a constant magnetic field $B$ decreases the potential energy in the true vacuum even in the curved spacetime. Curvature effects depend on the sign of the spacetime curvature $R$ as is shown in Fig. 2.(b). A negative curvature decreases the potential energy. On the contrary, positive curvature increases the potential energy. The broken chiral symmetry is restored for a sufficiently large and positive curvature even in an external large magnetic field.

\section{SUSY NJL model}

In the present section we consider the supersymmetric NJL model in the external constant magnetic field and external gravity.

In flat space such a model has been introduced in Ref. [22]. Generalization of the model for the case of non-minimal coupling with the external gravitational field (via scalar-gravitational coupling constants) has been presented in Ref. [23]. We consider the model of Ref. 223 in curved spacetime with the external magnetic field. The action in components is given by

$$
\begin{aligned}
S= & \int d^{4} x \sqrt{-g}\left[-\phi^{\dagger}\left(D^{\mu} D_{\mu}+\sigma^{2}+\xi_{1} R\right) \phi-\phi^{c \dagger}\left(D^{\mu} D_{\mu}+\sigma^{2}+\xi_{2} R\right) \phi^{c}\right. \\
& \left.+\bar{\psi}\left(i \gamma^{\mu} D_{\mu}-\sigma\right) \psi-\frac{1}{2 \lambda} \sigma^{2}\right]
\end{aligned}
$$

where $\sigma$ is an auxiliary scalar as in the original NJL model, $\psi$ is $N$ component Dirac spinor.

Note that actually the action (70) represents SUSY NJL model non-minimally interacting with the external gravity and minimally interacting with the external magnetic field. It is evident that supersymmetry of SUSY NJL model is always broken in the external fields. So it maybe natural to call the theory with action (70) the extended NJL model.

At the leading order of the $1 / N$ expansion the effective potential of the SUSY NJL model (70) is given by

$$
\begin{aligned}
& V_{e f f}(\sigma)=\frac{1}{2 \lambda} \sigma^{2}+i S p \ln \left\langle x\left|\left[i \gamma^{\mu}(x) D_{\mu}-\sigma\right]\right| x\right\rangle \\
& -i S p \ln \left\langle x\left|\left[D^{\mu} D_{\mu}+\sigma^{2}+\xi_{1} R\right]\right| x\right\rangle \\
& -i S p \ln \left\langle x\left|\left[D^{\mu} D_{\mu}+\sigma^{2}+\xi_{2} R\right]\right| x\right\rangle .
\end{aligned}
$$

The second term in the right hand side of Eq.(71) corresponds to the radiative correction by spinor fields and is rewritten by the spinor GF as is evaluated in the previous section (See Eq.(65)). The third and the fourth terms in the right hand side of Eq.(71) correspond to the radiative correction by scalar fields and is represented by the scalar GF

$$
\begin{array}{r}
-i S p \ln \left\langle x\left|\left[D^{\mu} D_{\mu}+\sigma^{2}+\xi R\right]\right| x\right\rangle \\
=2 i \int^{\sigma} m d m G(x, x, m) .
\end{array}
$$


Because of the supersymmetry both the radiative correction by spinor fields and the one by scalar fields are cancelled out in flat spacetime with vanishing electromagnetic fields. Thus the vacuum expectation value of $\sigma$ disappears for $R=B=0$. Substituting Eqs.(34) and (35) into Eq.(72) and integrating over $m$ one gets

$$
\begin{aligned}
& 2 i \int^{\sigma} m d m G(x, x, m) \\
& =-\frac{1}{(4 \pi)^{D / 2}} \int_{0}^{\infty} d s s^{-D / 2} \frac{e B}{\sinh (e B s)} \exp \left(-\sigma^{2} s\right) \\
& +\frac{1}{(4 \pi)^{D / 2}} \frac{R}{D(D-1)} \int_{0}^{\infty} \int_{0}^{\infty} d t d s \exp \left[-(s+t) \sigma^{2}\right] \\
& \times \frac{(s+t)^{-D / 2}}{1+e B t \operatorname{coth}(e B s)} \frac{e B}{\sinh (e B s)} \\
& \times\left\{\left[-\frac{D-3}{6}(D-2+2 e B s \operatorname{coth}(e B s))+\frac{-2 D+1}{3}\right] \frac{(D-2) t}{s+t}\right. \\
& +\left[-\frac{D-3}{6 s}(D-2+2 e B s \operatorname{coth}(e B s))+\frac{-2 D+1}{3} e B \operatorname{coth}(e B s)\right] \\
& \times \frac{2 t}{1+e B t \operatorname{coth}(e B s)} \\
& +\frac{2(D-1)}{3}\left[\frac{D(D-2)}{4}\left(\frac{t}{s+t}\right)^{2}+2\left(\frac{e B t \operatorname{coth}(e B s)}{1+e B t \operatorname{coth}(e B s)}\right)^{2}\right] \\
& +\frac{1}{3 s}\left[(D-3)\left((e B s)^{2} \operatorname{coth}^{2}(e B s)+1\right)+4 e B s \operatorname{coth}(e B s)\right] \\
& \left.\times \frac{(D-2) t}{s+t} \frac{t}{1+e B t \operatorname{coth}^{2}(e B s)}+D(D-1) \xi\right\} .
\end{aligned}
$$

In some special dimensions Eq.(73) simplifies. Inserting the Eq.(73) into Eq.(71) we obtain the final expression of the effective potential of the SUSY NJL model. Therefore the effective potential for $D=4$ reads

$$
\begin{aligned}
& V_{e f f}(\sigma)=\frac{1}{2 \lambda} \rho^{2}+\frac{1}{8 \pi^{2}} \int_{1 / \Lambda^{2}}^{\infty} \frac{d s}{s^{3}} \exp \left(-s \sigma^{2}\right) e B s \operatorname{coth}(e B s) \\
& -\frac{R}{192 \pi^{2}} \int_{1 / \Lambda^{2}}^{\infty} \int_{1 / \Lambda^{2}}^{\infty} \frac{d s d t}{(s+t)^{3}(1+e B t \operatorname{coth}(e B s))^{2}} \\
& \times \exp \left[-(s+t) \sigma^{2}\right][e B t(e B t+e B s) \\
& \left.+2(e B t+3 e B s) \operatorname{coth}(e B s)+2 e B t(e B s-e B t) \operatorname{coth}^{2}(e B s)\right] \\
& -\frac{1}{8 \pi^{2}} \int_{1 / \Lambda^{2}}^{\infty} \frac{d s}{s^{3}} \frac{e B s}{\sinh (e B s)} \exp \left(-s \sigma^{2}\right) \\
& +\frac{R}{48 \pi^{2}} \int_{1 / \Lambda^{2}}^{\infty} \int_{1 / \Lambda^{2}}^{\infty} \frac{d s d t}{(s+t)^{2}(1+e B t \operatorname{coth}(e B s))}
\end{aligned}
$$




$$
\begin{aligned}
& \times \frac{e B}{\sinh (e B s)} \exp \left[-(s+t) \sigma^{2}\right]\left\{\left[-\frac{4}{3}-\frac{1}{6} e B s \operatorname{coth}(e B s)\right] \frac{2 t}{s+t}\right. \\
& +\left[-\frac{1}{6 s}-\frac{4}{3} e B \operatorname{coth}(e B s)\right] \frac{2 t}{1+e B t \operatorname{coth}(e B s)} \\
& +2\left[\left(\frac{t}{s+t}\right)^{2}+\left(\frac{e B t \operatorname{coth}(e B s)}{1+e B t \operatorname{coth}(e B s)}\right)^{2}\right] \\
& +\frac{1}{3 s}\left[(e B s)^{2} \operatorname{coth}^{2}(e B s)+1+4 e B s \operatorname{coth}(e B s)\right] \\
& \left.\times \frac{t}{s+t} \frac{t}{1+e B t \operatorname{coth}(e B s)}+3\left(\xi_{1}+\xi_{2}\right)\right\}
\end{aligned}
$$

where we regularize the effective potential by introducing the proper-time cut-off $\Lambda$. The effective potential (74) reproduces the expression for $V_{\text {eff }}(\sigma)$ in Ref.23 at the limit $B \rightarrow 0$. The proper-time integrations in Eq.(74) are numerically performed below.

For $D=3$ the effective potential reads

$$
\begin{aligned}
& V_{e f f}(\sigma)=\frac{\sigma^{2}}{2 \lambda}+\frac{1}{4 \pi^{3 / 2}} \int_{1 / \Lambda^{2}}^{\infty} \frac{d s}{s^{5 / 2}} \exp \left(-s \sigma^{2}\right) e B s \operatorname{coth}(e B s) \\
& -\frac{R}{144 \pi^{3 / 2}} \int_{1 / \Lambda^{2}}^{\infty} \int_{1 / \Lambda^{2}}^{\infty} \frac{d s d t}{(t+s)^{5 / 2}(1+e B t \operatorname{coth}(e B s))^{2}} \\
& \times \exp \left[-(t+s) \sigma^{2}\right][2 e B t(e B t+e B s) \\
& \left.+(9 e B s+5 e B t) \operatorname{coth}(e B s)+e B t(e B s-3 e B t) \operatorname{coth}^{2}(e B s)\right] \\
& -\frac{1}{4 \pi^{3 / 2}} \int_{1 / \Lambda^{2}}^{\infty} \frac{d s}{s^{5 / 2}} \exp \left(-s \sigma^{2}\right) \frac{e B s}{\sinh (e B s)} \\
& +\frac{R}{24 \pi^{3 / 2}} \int_{1 / \Lambda^{2}}^{\infty} \int_{1 / \Lambda^{2}}^{\infty} \frac{d s d t}{(t+s)^{3 / 2}(1+e B t \operatorname{coth}(e B s))} \\
& \times \frac{e B}{\sinh (e B s)} \exp \left[-(s+t) \sigma^{2}\right]\left\{-\frac{5}{3} \frac{t}{s+t}-\frac{5}{3} \frac{2 e B t \operatorname{coth}(e B s)}{1+e B t \operatorname{coth}(e B s)}\right. \\
& +\left(\frac{t}{s+t}\right)^{2}+\frac{8}{3}\left(\frac{e B t \operatorname{coth}(e B s)}{1+e B t \operatorname{coth}(e B s)}\right)^{2} \\
& +\frac{t}{3} \frac{e B t \operatorname{coth}(e B s)}{s+t} \frac{\left.e B\left(\xi_{1}+\xi_{2}\right)\right\} .}{1+e B t \operatorname{coth}(e B s)}+3 .
\end{aligned}
$$

Since the contribution of scalar fields and fermion fields are canceled in a supersymmetric theory only a symmetric phase is realized for $R=B=0$. The external gravitational and magnetic fields considered here break the supersymmetry of the theory. Thus we expect that the chiral symmetry may be broken down. Using Eq.(74) we numerically calculate the effective potential of the SUSY NJL model for $D=4$ to study the phase structure of the theory. 
$V_{e f f}^{(0)}(\sigma) / \mu^{4}$

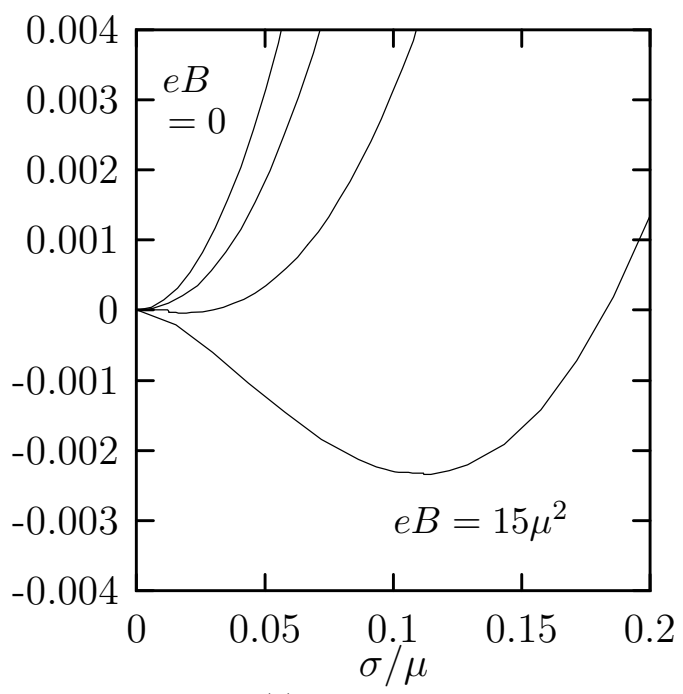

(a) $V_{\text {eff }}^{(0)}$ for $R=0$.
$V_{e f f}^{(1)}(\sigma) / \mu^{4}$

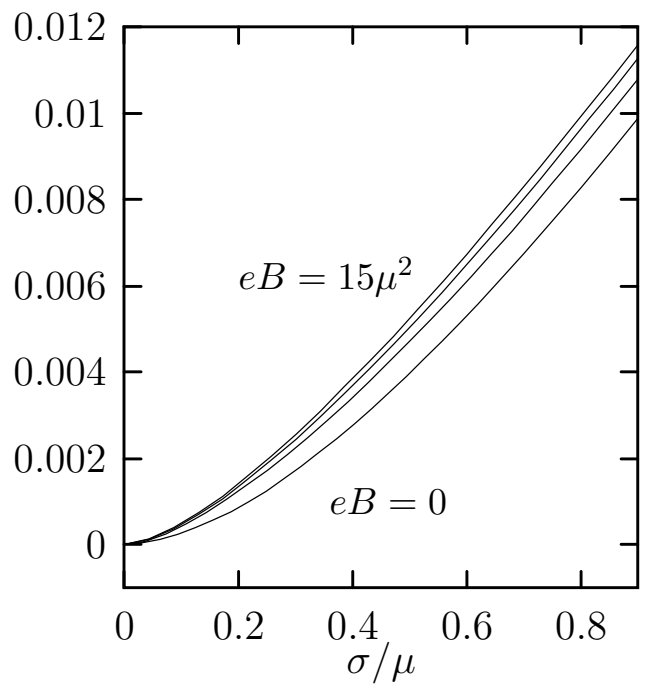

(b) $V_{e f f}^{(1)}$ for $R=0$ and $\xi_{1}+\xi_{2}=0$.

$V_{e f f}^{(1)}(\sigma) / \mu^{4}$

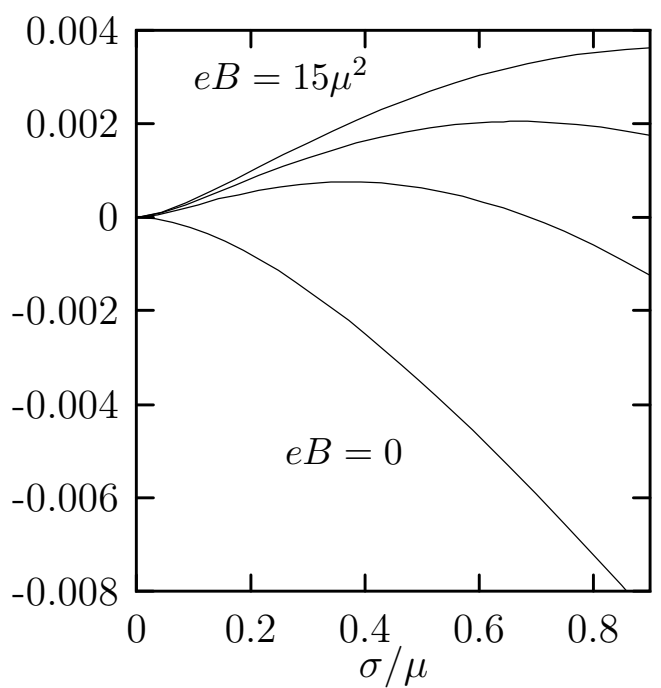

(c) $V_{\text {eff }}^{(1)}$ for $R=0$ and $\xi_{1}+\xi_{2}=1$.

Figure 3: The behavior of the effective potential $V_{\text {eff }}^{(0)}$ and $V_{e f f}^{(1)}$ are shown with the varying magnetic field $e B\left(=0,5 \mu^{2}, 10 \mu^{2}, 15 \mu^{2}\right)$ for fixed $\lambda(=1 / 2.5)$, and fixed $\Lambda(=10 \mu)$ in four dimensions. $\mu$ is an arbitrary mass scale. We normalize that $V_{e f f}(0)=0$. 
$V_{e f f}(\sigma) / \mu^{4}$

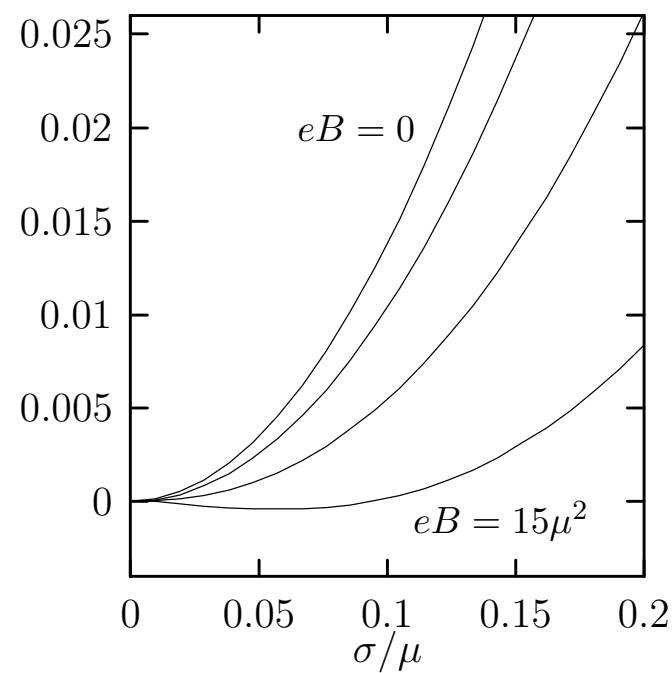

(a) $V_{\text {eff }}$ for $R=5 \mu^{2}$ and $\xi_{1}+\xi_{2}=0$.

$V_{e f f}(\sigma) / \mu^{4}$

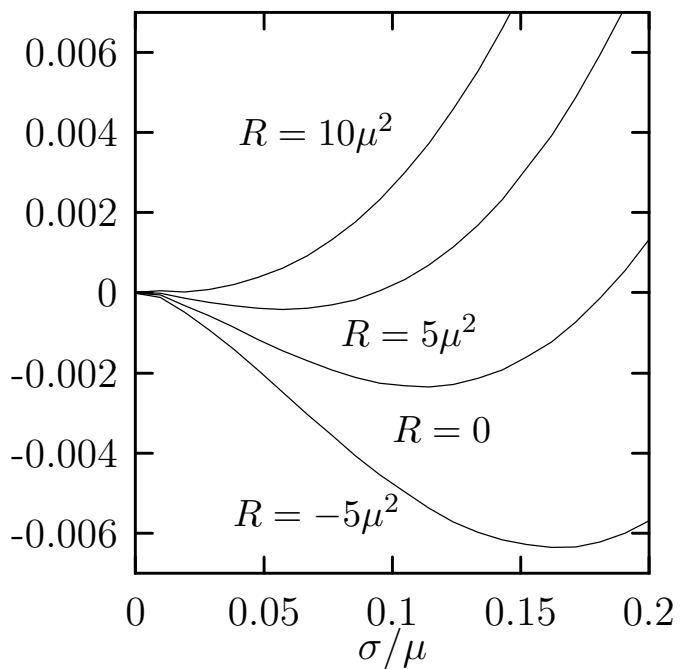

(c) $V_{\text {eff }}$ for $e B=15 \mu^{2}$ and $\xi_{1}+\xi_{2}=0$.
$V_{e f f}(\sigma) / \mu^{4}$

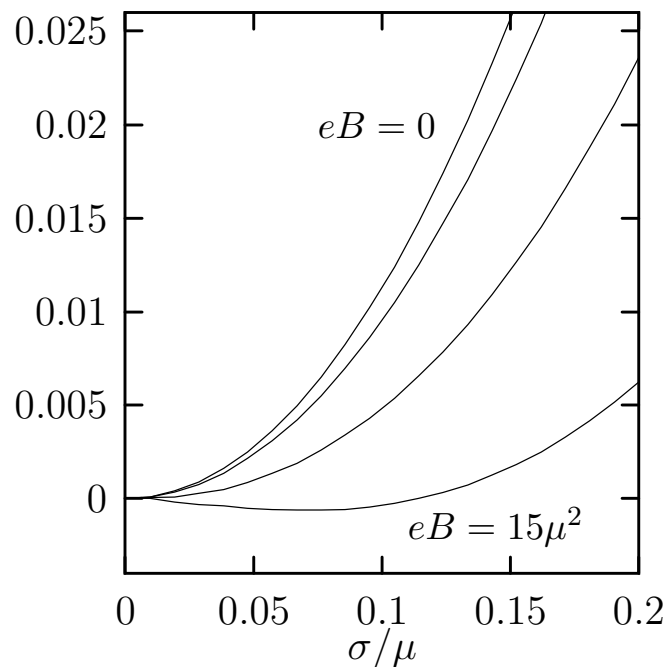

(b) $V_{\text {eff }}$ for $R=5 \mu^{2}$ and $\xi_{1}+\xi_{2}=1$.

$V_{\text {eff }}(\sigma) / \mu^{4}$

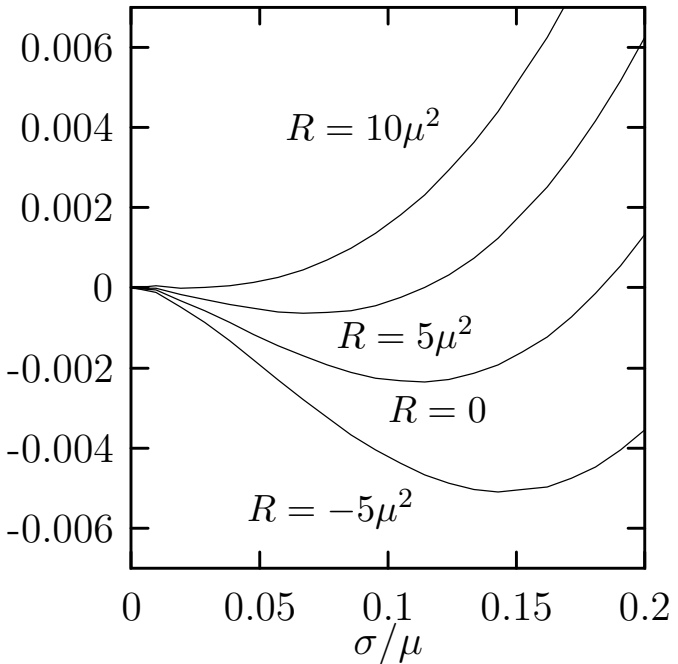

(d) $V_{\text {eff }}$ for $e B=15 \mu^{2}$ and $\xi_{1}+\xi_{2}=1$.

Figure 4: The behavior of the effective potential $V_{\text {eff }}$ are shown with the varying $B$ or $R$ for fixed $\lambda(=1 / 2.5)$, and fixed $\Lambda(=10 \mu)$ in four dimensions. 
In Figs. 3 and 4 we illustrate the typical behaviors of the effective potential for $D=4$ in the SUSY NJL model. In Fig.3 we draw the terms independent of $\mathrm{R}, V^{(0)}$, and the linear curvature correction to the effective potential, $V^{(1)}$. The linear curvature correction strongly depends on the coupling constant $\xi_{1}+\xi_{2}$. There are large cancellations between the corrections of fermion and scalar fields. Thus the terms of the linear curvature correction in the SUSY NJL model are significally smaller than that in the NJL model. As is clearly seen in Fig.4 the chiral symmetry is broken down for a sufficiently large magnetic field and/or a negative curvature for $R=5 \mu^{2}$ or $B=15 \mu^{2}$. The external curvature has the opposite effects in the case of large $\xi_{1}+\xi_{2}(>1 / 2)$ for a small $B$ and/or large $\sigma$ (See Fig.3.(c)).

\section{Conclusion}

In the present paper we discussed the combined effect of the gravitational and magnetic fields to the chiral symmetry breaking in NJL and SUSY NJL models. Chiral symmetry is broken at non- zero and non- positive curvature. On the same time, positive curvature acts against chiral symmetry breaking. Nevertheless, the magnetic field effects may be significally stronger in realistic situations corresponding to early Universe with primordial magnetic fields.

It should be noted that the effective potential calculated in the present paper depends on how to introduce cut-off parameter in the proper-time integral, though we do not develop it any further here.

Using the results presented in Ref. [5] it is not difficult to calculate the effective potential in above two models exactly on the constant curvature spacetimes (DeSitter or anti- DeSitter, for example [5, 7]). Such calculation shows that curvature effects estimated in this work are taken into account qualitatively correctly (one can consider then very strong curvature as well). However it is not clear how to make such calculation exactly both on curvature and on magnetic field. The only possibility to do so is to work on some gravitational- magnetic background where exact solutions of field equations are known (for example, on conformal spacetime with magnetic field).

Another interesting proposal could be to study the dynamical symmetry breaking on electromagnetic- gravitational background representing a combination of constant electromagnetic field with gravitational wave (it is known the effective Lagrangian in pure electromagnetic or gravitational wave is trivial and no effect is expected). Such background may be roughly considered as signal which comes from strongly- graviting object with strong magnetic field. Then one may speculate on the possible use of the dynamical symmetry breaking in gravitational waves detectors.

We thank I. L. Buchbinder, S. J. Gates and T. Muta for helpful discussions. T. I. was supported in part by Monbusho Grant-in-Aid for Scientific Research Fellowship, No.2616. S. D. O. thanks COLCIENCIES (Colombis) and JSPS (Japan) for partial support of this 
work. This work (Yu. I. Sh. and S. D. O.) was supported in part by Ministerio de Educacion y Cultura (Spain). Yu. I. Sh. also expresses his deep gratitude to A. Letwin and R. Patov for their kind support.

\section{References}

[1] Y. Nambu and G. Jona-Lasinio, Phys. Rev. 122, (1961) 345.

[2] D. Gross and A. Neveu, Phys. Rev. D10, (1974) 3235.

[3] T. Inagaki, T. Muta and S. D. Odintsov, Mod. Phys. Lett., A8, (1993) 2117.

[4] E. Elizalde, S. D. Odintsov and Yu .I. Shil'nov, Mod. Phys. Lett. A9, (1994) 913.

[5] T. Inagaki, T. Muta and S. D. Odintsov, Dynamical Symmetry Breaking in Curved Spacetime, to be published in Prog. Theor. Phys. (Supplment).

[6] T. Inagaki, Int. J. Mod. Phys. A11 (1996), 4561.

[7] T. Inagaki, S.Mukaigawa and T.Muta, Phys. Rev. D52, (1996) R4267;

E. Elizalde, S. Leseduarte, S. D. Odintsov and Yu. I. Shil'nov, Phys. Rev. D53, (1996) 1917;

K. Ishikawa, T. Inagaki and T.Muta, Mod. Phy. Lett A11, (1996) 939.

T. Inagaki and K. Ishikawa, Thermal and Curvature Effects to the Dynamical Symmetry Breaking, to be published in Phys. Rev. D. G. Miele and P. Vitale, Nucl. Phys., B494 (1997), 365

[8] T. Muta and S. D. Odintsov, Mod. Phys. Lett. A6, (1991) 3641;

C. T. Hill and D. S. Salopek, Ann. Phys. (NY). 213, (1992) 21.

[9] M. S.Turner and L. M. Widrow, Phys. Rev. D37, (1988) 2743;

T. Vachaspati, Phys. Lett. B263, (1991) 258;

K. Enqvist and P. Olesen, Phys. Lett. B319, (1993) 178;

M. Gasperini, M. Giovannini and G. Veneziano, Phys. Rev. Lett. 75, (1995) 3796;

G. Baum, D. Bodeker and L. McLerran, Phys. Rev. D53, (1996) 662;

J. M. Cornwall, in Unified Symmetry, ( ed. by B. N. Kursunoglu et. al., Plenum, N.Y., 1995.)

[10] S. P. Klevansky and R. H. Lemmer, Phys. Rev. D38, (1988) 3559;

H. Suganuma and T. Tatsumi, Ann. of Phys. (N.Y.) 208, (1991) 470; Progr. Theor. Phys. 90, (1993) 379;

I. Krive and S. Naftulin, Phys. Rev. D46, (1992) 2337;

S. P. Klevansky, Rev. Mod. Phys. 64, (1992) 1;

D. Cangemi, E. D.'Hoker and G. V. Dunne, Phys. Rev. D51, (1995) 2513. 
[11] K. G. Klimenko, Theor. Math. Phys. (in Russinan) 89 (1991), 211;

Z. Phys. C54, (1992) 323;

A. S. Vshivtsev, B. V. Magnitski and K. G. Klimenko, JETP Lett. 62 (1995) 283.

[12] C. N. Leung, Y. J. Ng and A. W. Ackly, Phys. Rev. D54, (1996), 4181;

D.- S. Lee, C. N. Leung, Y. J. Ng, hep- th/9701172;

M. Ishi- i, T. Kashiwa and N. Tanemura, hep- th/9707248;

S. Kanemura, H. Sato and H. Tochimura, hep- th/9707285

[13] D. M. Gitman, S. D. Odintsov and Yu. I. Shil'nov, Phys. Rev. D54, (1996) 2968.

[14] B. Geyer, L. N. Granda and S. D. Odintsov, Mod. Phys. Lett. A11, (1996) 2053. I. Brevik, D. M. Gitman, S. D. Odintsov, hep-th/9611138; Gravitation and Cosmology, to appear

[15] T. S. Bunch and L. Parker, Phys. Rev. D20, (1979) 2449.

[16] A. Z. Petrov, Einstein Spaces (Pergamon, Oxford, 1969).

[17] L. Parker and D. Toms, Phys. Rev. D29, (1984) 1584.

[18] J. Schwinger, Phys. Rev. 82, (1951) 664.

[19] C. Itzykson and J.-B. Zuber, "Quantum Field Theory", (McGraw-Hill Inc., 1980).

[20] V. G. Bagrov and D. M. Gitman, Exact Solution of Relativistic Wave Equation, (Kluwer Ac. Pub. Dorolrecht, 1990.)

[21] J. M. Cornwall, R. Jackiw and E. Tomboulis, Phys. Rev., D10, (1974) 2428.

[22] W. Buchmuller and S. T. Love, Nucl. Phys. B204, (1982) 213.

[23] I. L. Buchbinder, T. Inagaki and S. D. Odintsov, hep-th/9702097.

[24] I. L. Buchbinder, S. D. Odintsov, I. L. Shapiro, Effective Action in Quantum Gravity, (IOP Publishing, Bristol and Philadelphia, 1992.) 